Proceedings of the Edinburgh Mathematical Society (2002) 45, 717-721 (C)

DOI:10.1017/S0013091501000311 Printed in the United Kingdom

\title{
ON LOCAL FINITENESS OF PERIODIC RESIDUALLY FINITE GROUPS
}

\author{
MAHMUT KUZUCUOĞLU ${ }^{1}$ AND PAVEL SHUMYATSKY ${ }^{2}$ \\ ${ }^{1}$ Department of Mathematics, Middle East Technical University, \\ 06531 Ankara, Turkey (matmah@metu.edu.tr) \\ ${ }^{2}$ Department of Mathematics, University of Brasilia, Brasilia-DF, \\ 70910-900 Brazil (pavel@ipe.mat.unb.br)
}

(Received 13 March 2001)

Abstract Let $G$ be a periodic residually finite group containing a nilpotent subgroup $A$ such that $C_{G}(A)$ is finite. We show that if $\left\langle A, A^{g}\right\rangle$ is finite for any $g \in G$, then $G$ is locally finite.

Keywords: automorphisms; centralizers; periodic groups

AMS 2000 Mathematics subject classification: Primary 20F50

\section{Introduction}

It is now well known that periodic groups need not be locally finite. Examples constructed in $[4-6,11]$ show that there even exist periodic residually finite groups that are not locally finite. On the other hand, following Zel'manov's solution of the restricted Burnside problem $[\mathbf{1 2}, \mathbf{1 3}]$, certain useful theorems on local finiteness of such groups have recently been established. The main goal of the present paper is to prove the following result.

Theorem 1.1. Let $G$ be a periodic residually finite group containing a nilpotent subgroup $A$ such that $C_{G}(A)$ is finite. Assume that $\left\langle A, A^{g}\right\rangle$ is finite for any $g \in G$. Then $G$ is locally finite.

The above theorem essentially belongs to the same type of result as those obtained in Shalev $[\mathbf{8}]$ and Shumyatsky $[\mathbf{9}]$. In fact, Shalev's results show that in the case in which $A$ is a finite 2-group, the hypothesis that $\left\langle A, A^{g}\right\rangle$ is finite for any $g \in G$ can be omitted (if $A$ is of order 2, then, by virtue of Shunkov's theorem [10], even the assumption that $G$ is residually finite is not required). It seems, however, that the 2-group case is special and in general the hypothesis on $\left\langle A, A^{g}\right\rangle$, or something similar, is necessary. We also mention that Golod's example [4] shows that we cannot drop out the hypothesis that $C_{G}(A)$ is finite.

The main idea of the proof of Theorem 1.1 is to use a powerful Lie-theoretic criterion for a finitely generated residually finite group to be finite, discovered by Zel'manov [14, Theorem 1.6], combining it with a theorem of Bahturin and Zaicev on polynomial identities 
in graded Lie algebras [1]. The reduction of the theorem to a Lie-theoretic problem is performed using some deep results of Hartley on automorphisms of finite groups. Since Hartley's results have not been published, their full proofs are included in the next section.

\section{Finite groups with a fixed-point-free nilpotent group of automorphisms}

We say that $A$ acts fixed-point-freely on $G$, if $C_{G}(A)=1$. The main goal of this section is to prove the following theorem.

Theorem 2.1 (Hartley). If a finite nilpotent group $A$ acts fixed-point-freely on a finite group $G$, then $G$ is soluble.

The following lemmas will be useful.

Lemma 2.2. Let $q$ be a prime and let $A$ be a finite q-group acting fixed-point-freely on a finite group $G$. Then $G$ is soluble.

Proof. It is a well-known corollary of the classification of finite simple groups that if a finite group $G$ admits a coprime fixed-point-free group of automorphisms, then $G$ is soluble. However if $|G|$ is divisible by $q$, then $A$ fixes an element of order $q$.

Lemma 2.3. Let $A$ be a finite nilpotent group acting on a finite group $G$, and suppose that $C_{G}(A)=1$. Let $N$ be a soluble normal $A$-invariant subgroup of $G$. Then $C_{G / N}(A)=1$.

Proof. If $C_{G / N}(A) \neq 1$, then there exists a non-trivial cyclic subgroup $H / N$ of $G / N$ such that $A$ acts trivially on $H / N$. Then $H$ is a soluble group, and $A$ normalizes it. We have $N_{H A}(A)=A$, and so $A$ is a Carter subgroup of $H A$. Therefore, $N A / N$ is a Carter subgroup of $H A / N$ and since it is normal in $H A / N$, we find that $N A / N=H A / N$, hence $H=N$, a contradiction.

Proof of Theorem 2.1. Let $G$ be a minimal counterexample to the theorem. Then it follows from Lemma 2.3 that the soluble radical of $G$ is trivial, and then, by considering a minimal normal subgroup of $G A$, that $G$ is a direct product of copies of a simple nonabelian group permuted transitively by $A$. Write $G=S_{1} \times \cdots \times S_{n}$, and let $B=N_{A}\left(S_{1}\right)$. Then $B$ is nilpotent and acts fixed-point-freely on $S_{1}$, hence we obtain $S_{1}=G$. Thus we have that $G$ is non-abelian simple. We think of $G$ as a normal subgroup of $J=\operatorname{Aut}(G)$, and so $A \leqslant J$. If $A \cap G \neq 1$, then since $A \cap G$ is normal in $A$ we have $Z(A) \cap G \neq 1$, a contradiction. So $A \cap G=1$. If $G$ is sporadic or alternating, then $|J / G|$ is a power of 2 . So by Lemma 2.2 we get a contradiction.

Hence $G$ is of Lie type. We think of $G$ as embedded in a simple algebraic group of adjoint type $\bar{G}$ in the usual way. More precisely, we have a Frobenius map $\sigma$ on $\bar{G}$, and $G=O^{p^{\prime}}\left(\bar{G}_{\sigma}\right)$. In what follows we use the notation of $[7]$. Thus

$$
J=\bar{G}_{\sigma} \Phi_{G} \Gamma_{G} .
$$


We prove by induction on $|G|$ that under these assumptions $C_{G}(A) \neq 1$. Consider again a minimal counterexample to this statement. Let $A=A_{1} \times \cdots \times A_{s}$, where the factors are the non-trivial Sylow subgroups of $A$. For each $i$ choose a subgroup $Z_{i}$ of prime order in $Z\left(A_{i}\right)$. Suppose first that $Z_{i} \cap \bar{G}_{\sigma}=1$ for some $i$. Let $C=C_{G}\left(Z_{i}\right)$. Then by [7, Lemma 3.1] $C$ is insoluble. On the other hand, $A$ acts on $C$. So by the induction assumption we get a contradiction.

So we assume that $Z_{i} \cap \bar{G}_{\sigma} \neq 1$ for all $i$, while $A \cap G=1$. This means that every prime divisor of $|A|$ also divides $\left|\bar{G}_{\sigma} / G\right|$, which is the group of diagonal automorphisms. It follows that the order of $A$ is prime to the characteristic of the field over which $\bar{G}$ is defined. Now $\bar{G}_{\sigma} / G$ is a 2 -group or a 3 -group except in the case when $\bar{G}$ is of type $\boldsymbol{A}$. So, except in this case, $A$ is a 2 -group or a 3 -group and by Lemma 2.2 we get a contradiction. Therefore $\bar{G}$ is of type $\boldsymbol{A}$. Every prime divisor of $\left|\bar{G}_{\sigma} / G\right|$ also divides $|G|$. It follows from Lemma 2.2 that $|A|$ is divisible by at least two distinct primes $q$ and $r$. Let $Z$ be a central subgroup of order $q$ in $A$. So we have $\bar{G}=P G L_{n}(K)$, where $K$ is some algebraically closed field of characteristic $p$ distinct from $q$ and $r$. Thus $\left|\bar{G}_{\sigma} / G\right|$ divides $n$, and so $q r \mid n$, hence

$$
q<n
$$

Let $D=C_{\bar{G}}(Z)$. Then by $[\mathbf{7}$, Lemma 3.4$]$ the group $C_{G}(Z)=O^{p^{\prime}}\left(D_{\sigma}^{0}\right)$, involves a smaller simple group of the same type as $G$ which is a contradiction. This concludes the proof.

Corollary 2.4. Let $A$ be a finite group. Then there exists a finite insoluble group $G$ and a fixed-point-free action of $A$ on $G$, if and only if $A$ is not nilpotent.

Proof. If $A$ is not nilpotent, then the existence of $G$ follows from [2]. The converse is clear from the above theorem.

\section{Main result}

Lemma 3.1. Let $G$ be a periodic group acted on by a nilpotent group $A$ such that $C_{G}(A)=1$. Suppose that $\left\langle A, A^{g}\right\rangle$ is finite for any $g \in G$. Assume $N$ is an $A$-invariant normal subgroup of $G$. Then $C_{G / N}(A)=1$.

Proof. Assume $C_{G / N}(A) \neq 1$ and choose a non-trivial element $x N \in C_{G / N}(A)$. Consider the subgroup $H$ generated by $x$ and all elements of the form $x^{-1} x^{a}$, where $a$ ranges through $A$. Then $H$ is a finite $A$-invariant subgroup of $G$. By Theorem 2.1 $H$ is soluble and so, by Lemma $2.3, C_{H / H \cap N}(A)=1$. This yields a contradiction since $x(H \cap N)$ obviously lies in $C_{H / H \cap N}(A)$.

Given a finite group $G$, the Fitting subgroup of $G$ will be denoted, as usual, by $F(G)$. By induction we set $F_{0}(G)=1$ and $F_{i+1}(G) / F_{i}(G)=F\left(G / F_{i}\right)$ for $i \geqslant 0$. Recall that if $G$ is soluble, the Fitting height $h(G)$ of $G$ is defined as the least number $h$ such that $G=F_{h}(G)$.

Lemma 3.2. Let $G$ be a periodic group acted on by a nilpotent group $A$ such that $C_{G}(A)=1$. Suppose that $\left\langle A, A^{g}\right\rangle$ is finite for any $g \in G$. Let $R$ be any finite $A$-invariant 
section of $G$. Then $R$ is soluble and $h(R)$ is bounded by a function depending only on the order of $A$.

Proof. It follows from Lemma 3.1 that $C_{R}(A)=1$ and, therefore, by Theorem $2.1, R$ is soluble. Hence $A$ is a Carter subgroup of the finite soluble group $R A$. It is a well-known result of Dade that the Fitting height of a finite soluble group is bounded by a function depending only on the order of a Carter subgroup [3].

Lemma 3.3. Let $p$ be a prime. Let $G$ be a finitely generated periodic residually finite p-group acted on by a nilpotent group $A$ such that $C_{G}(A)=1$. Suppose that $\left\langle A, A^{g}\right\rangle$ is finite for any $g \in G$. Then $G$ is finite.

Proof. Let $B$ be the maximal $p^{\prime}$-subgroup of $A$. It is easy to see that $C_{G}(B)=1$. Thus, replacing $A$ by $B$ we can assume that $A$ is a $p^{\prime}$-group. Let $D_{i}$ denote the $i$ th term of the Lazard series of $G$, and let $L_{p}(G)$ be the corresponding Lie algebra constructed as in Zel'manov [14]. In a natural way, the group $A$ acts on each quotient $D_{i} / D_{i+1}$. This induces an action of $A$ on $L_{p}(G)$ and so we can view $A$ as an automorphism group of $L_{p}(G)$. Lemma 3.1 shows that $A$ is fixed-point-free on each quotient $D_{i} / D_{i+1}$ and so we deduce that 0 is the only fixed-point of $A$ in $L_{p}(G)$. Since $A$ is a $p^{\prime}$-group, the theorem of Bahturin and Zaicev [1] guarantees that $L_{p}(G)$ satisfies a polynomial identity. Thus, finiteness of $G$ follows by Theorem 1.6 in Zel'manov [14].

Proof of Theorem 1.1. Since $G$ is residually finite, we can choose a normal subgroup $H$ of finite index such that $H \cap C_{G}(A)=1$. Of course, it is sufficient to show that $H$ is locally finite. We regard $A$ as a fixed-point-free group of automorphisms of $H$. By Lemma 3.2, any $A$-invariant finite quotient of $H$ is soluble and has Fitting height bounded by some function that depends only on the order of $A$. It follows that $H$ possesses a characteristic series of finite length $H=H_{1} \geqslant H_{2} \geqslant \cdots \geqslant H_{s}=1$ all of whose quotients are residually finite-nilpotent. Arguing by induction on $s$ we can assume that $H$ is residually nilpotent. Then $H$ is a direct product of its Sylow $p$-subgroups and so without any loss of generality we can assume that $H$ is a $p$-group for some prime $p$. Since any finite subset of $H$ is contained in a finitely generated $A$-invariant subgroup, local finiteness of $H$ is immediate from Lemma 3.3.

Acknowledgements. P.S. was supported by FAPDF and TUBITAK.

\section{References}

1. Yu. A. Bahturin And M. V. Zaicev, Identities of graded algebras, J. Alg. 205 (1998), $1-12$.

2. S. D. Bell and B. Hartley, A note on fixed-point free actions of finite groups, $Q . J$. Math. (2) 41 (1990), 127-130.

3. E. C. DADE, Carter subgroups and Fitting heights of finite solvable groups, Illinois J. Math. 13 (1969), 449-514.

4. E. S. Golod, On nil-algebras and residually finite groups, Izv. Akad. Nauk SSSR Ser. Mat. 28 (1964), 273-276. 
5. R. I. Grigorchuk, On the Burnside problem for periodic groups, Funct. Analysis Applic. 14 (1980), 53-54.

6. N. Gupta And S. Sidki, On the Burnside problem for periodic groups, Math. Z. 182 (1983), 385-386.

7. B. HARTLEy, A general Brauer-Fowler theorem and centralizers in locally finite groups, Pac. J. Math. 152 (1992), 101-117.

8. A. Shalev, Centralizers in residually finite torsion groups, Proc. Am. Math. Soc. 126 (1998), 3495-3499.

9. P. Shumyatsky, Centralizers in groups with finiteness conditions, J. Group Theory 1 (1998), 275-282.

10. V. P. Shunkov, On periodic groups with an almost regular involution, Alg. Logika 11 (1972), 470-493 (in Russian) (English transl.: Alg. Logic 11 (1972), 260-272).

11. V. I. Sushchansky, Periodic $p$-elements of permutations and the general Burnside problem, Dokl. Akad. Nauk SSSR 247 (1979), 447-461.

12. E. ZEL'MANOV, The solution of the restricted Burnside problem for groups of odd exponent, Math. USSR Izv. 36 (1991), 41-60.

13. E. ZEL'MANOv, The solution of the restricted Burnside problem for 2-groups, Mat. USSR Sb. 182 (1991), 568-592.

14. E. ZEL'MANOV, Lie ring methods in the theory of nilpotent groups, in Groups '93, GalwaySt Andrews, London Mathematical Society Lecture Note Series, vol. 212, pp. 567-586, (1995). 